\title{
Data Acquisition Software for a Prototype of LET Spectrometer
}

\section{Wenrui Sun, ${ }^{a, b, *}$ Zhe Cao, ${ }^{a, b, r}$ Li Wang, ${ }^{c}$ Changqing Feng, ${ }^{a, b}$ Chunjuan Li, ${ }^{d}$ Li Xia, ${ }^{d}$ Lei Zhao ${ }^{a, b}$ and Qi An ${ }^{a, b}$}

${ }^{a}$ State Key Laboratory of Particle Detection and Electronics, University of Science and Technology of China, No.96, JinZhai Road, Hefei, China

${ }^{b}$ Department of Modern Physics, University of Science and Technology of China, No.96, JinZhai Road, Hefei, China

${ }^{c}$ School of Earth and Space Sciences, University of Science and Technology of China, No.96 JinZhai Road, Hefei, China

${ }^{d}$ National Key Laboratory for Metrology and Calibration Techniques, China Institute of Atomic Energy, 1 Sanqiang Road, Beijing, China

${ }^{\dagger}$ Corresponding author

E-mail: caozhedustc.edu.cn

Silicon Strip Detector (SSD) has been widely used in detecting charged particles. Using SSD as detector, and to measure the Linear Energy Transfer (LET) generated by the ionizing radiation in manned spacecraft, a prototype of LET spectrometer is designed. This paper presents the design of the data acquisition (DAQ) software for the LET spectrometer. To read out and preliminarily analyze the data, the DAQ software is consist of three modules, which are readout and control module, data real-time imaging module, and offline data analysis module. The DAQ software realizes the dual communication with the electronics of the prototype through the Ethernet interface and the USB interface. Qt is used as the development tool for Windows platform, because of its excellent reliability and maintainability. $\mathrm{C}++$ is used as the programming language. In a priliminary test, the DAQ software shows good performance and fits the needs of application.

37th International Cosmic Ray Conference (ICRC 2021)

July 12 th $-23 \mathrm{rd}, 2021$

Online-Berlin, Germany

\section{"Presenter}




\section{Introduction}

In manned space missions, the ionizing radiation environment poses a hazard for both spacecraft and space crews, and the ionizing particle environment in space is the dominant source of ionizing radiation within space systems [1]. Solar energy particles (SEPs), galactic cosmic rays (GCRs), and trapped radiation are the main sources of space radiation. Charged particles are the main components of them. SEPs are consist of $92 \%$ protons, $6 \%$ helium ions, and $2 \%$ high-charge and high-energy particles (HZE); GCRs are consist of 85-90\% protons, $10-13 \%$ helium ions about $1 \%$ electrons, and 1\% HZEs; trapped radiation mainly consists of protons and electrons [2]. Measuring linear energy transfer (LET) of charged particles is a method to evaluate the risk of ionizing radiation [3] in space.

Aiming to measure LET of charged particles, a prototype of LET spectrometer is designed. Figure 1 is the schematic diagram of the prototype. The prototype uses double-sided silicon strip detectors (DSSD), which are a type of SSD and can obtain the incident position of particles from both of its sides, which are P-side and N-side, as the detectors. The DSSD used in the spectrometer has a total of 32 readout strips, 16 strips for each side. There are $\mathrm{N}$ detectors of the same specification in the prototype. The electronics of the prototype provides two different gains, corresponding to two different ranges. As a result, data generated by 32 strips of one detector end up in 64 valid channels of data. Due to the design of the chips used in the electronics, there are 64 invalid channels in the data. There is a correspondence between channels and the strips, gains and their validities. The data generated by the electronics are transferred to the upper computer through Ethernet or USB 2.0. To read out and preliminarily analyze the data, a DAQ software is designed and is proposed in this paper.

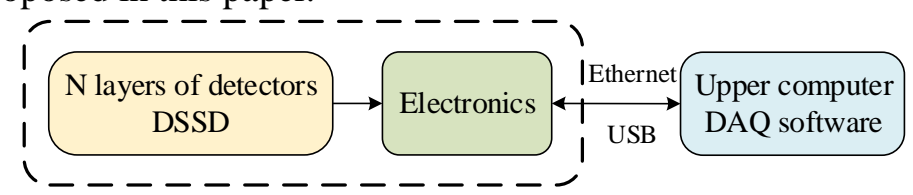

Figure 1: Schematic diagram of the prototype.

Data-based energy spectrums and hit position maps are shown on the software, which can be a tool for both prototype status check and data check. The algorithm that is used to calculate LET of given kinds of particles is also realized in the software. The energy spectrums and hit position maps can be acquired from data of each detector, while the LET spectrum needs more than two layers of detectors to be operating. In ways of connection, the Ethernet way is used to receive data generated by one or more detectors. The universal serial bus (USB) way can only receive data from one detector, but can be configured easily and is used for debugging.

In this paper, the implementation of the DAQ software is introduced in section 2. Section 3 introduces a preliminary test of the software. Section 4 concludes this paper.

\section{Software Implementation}

\subsection{Software Structure}

The DAQ software is developed with Qt framework and is designed to run on Windows platform. The Qt framework contains a comprehensive set of C++ library classes and produces codes with high runtime performance and small footprint [4]. To make software operation simple, 
a graphic user interface (GUI) is designed. As Figure 2 shows, the software adopts a Model-ViewController (MVC) structure. MVC is a design pattern used to decouple user-interface (view), data (model), and application logic (controller)[5].

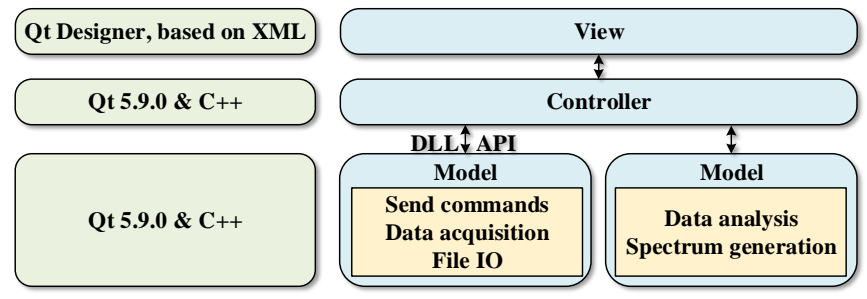

Figure 2: MVC structure of the software.

\subsubsection{View}

The view layer is designed with Qt Designer. The UI interface is stored in an extensible markup language (XML) file. The XML is a subset of standard generalized markup language [6] and is suitable to store the GUI tree [7]. On this layer, users' operations are converted to messages, which are sent to the controller layer. The results of the program are also displayed on this layer.

\subsubsection{Controller}

The controller layer is designed with Qt 5.9.0 and C++. It receives messages from the view layer and emits signals to the model layer. The results are sent to the view layer through the controller layer. It controls the performance of another two layers.

\subsubsection{Model}

The model layer includes the concrete realization of the functions and is written with Qt 5.9.0 and $\mathrm{C}++$. The function modules are introduced as below.

\subsection{Function modules}

There are three function modules in the DAQ software, which are readout and control module, data real-time imaging module, and offline data analysis module. The procedure flow chart is shown in Figure 3. In this figure, the readout and control module is in the green dashed lines; the data real-time imaging module is in the blue dashed lines; the offline data analysis module is in the red dashed lines.

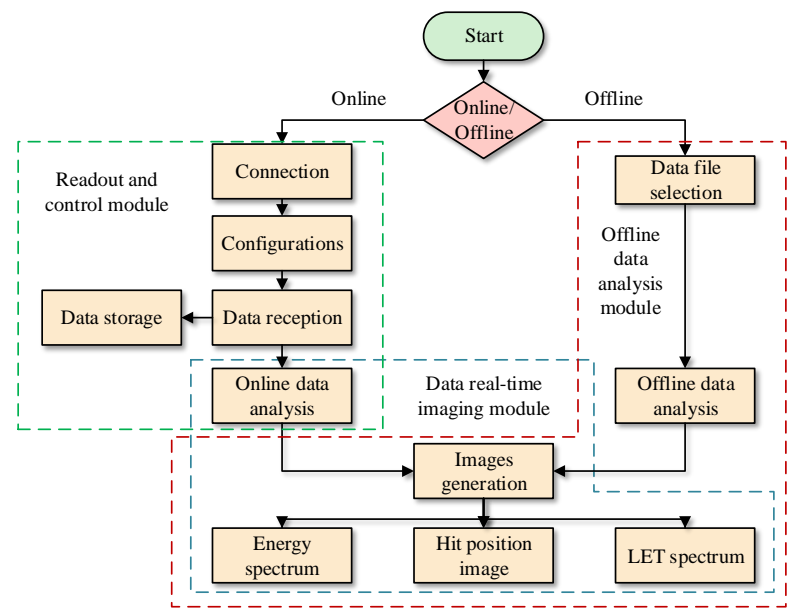

Figure 3: Procedure flow chart of the DAQ software 


\subsubsection{Readout and control module}

The readout and control module receives the data and sends commands through Ethernet or USB 2.0. To realize these functions, the upper computer needs to be connected with the electronics. The connection status will be shown on the software. To connect the upper computer with electronics through Ethernet, the user needs to configure the IP address and port number. The USB way of connection is designed for debugging. The VID and PID of the electronics' USB interface are fixed so that the users do not need extra configuration. After the hardware is connected, the software allows the users to configure the electronics. The details are shown in the GUI in subsection 2.5.

Raw data that are received by the upper computer are read into the random-access memory (RAM) and then stored on the hard disk. Data receiving and processing are carried out simultaneously, which are executed in different computer processes. This is implemented using QThread. The data format is shown in Table 1. In this table, $\mathrm{n}$ is the number of data messages in one package, and it depends on the electronics. The data is received asynchronously. The maximum rate of the data generated by the electronics is below $6 \mathrm{Mbit} / \mathrm{s}$, and there are 3 layers of electronics. As a result, the data receiving rate of the upper computer will not exceed $18 \mathrm{Mbit} / \mathrm{s}$. Low data rate makes it possible to process data in real-time.

Table 1: Data format in one package

\begin{tabular}{|c|c|c|}
\hline Name & Description & Length \\
\hline Package Head & Begin of a package & 2 bytes \\
\hline \multirow[t]{4}{*}{ Data } & $\begin{array}{l}\text { Data }_{1} \text { : Data of } 64 \text { channels with high gain and data of } 64 \\
\text { channels with low gain. The data of each channel is the } \\
\text { ADC value of the channel, arranged in the order of channel } \\
1 \text { to channel } 64 \text {, The data of high gain comes before the data } \\
\text { of low gain. }\end{array}$ & $2 \times 64 \times 2$ bytes \\
\hline & $\operatorname{Data}_{2}$ & $2 \times 64 \times 2$ bytes \\
\hline & $\ldots$ & $2 \times 64 \times 2 \times(n-3)$ bytes \\
\hline & Data $_{n}$ & $2 \times 64 \times 2$ bytes \\
\hline ID & Chip ID: The detector messages of the data. & 2 bytes \\
\hline Package tail & End of a package & 2 bytes \\
\hline Total & & $(256 \times n+6)$ bytes \\
\hline
\end{tabular}

\subsubsection{Data real-time imaging module}

Data real-time imaging module realizes online data analysis and real-time image rendering. After the data are received and stored in a hard disk, as subsection 2.2.1 shows, the data in RAM will be offered to the part of data analysis. In this part, the data of both axes of the images are determined. The first step is to check the integrity of the package of data. Packages that do not meet the format shown in Table 1 will be discarded in the process. Then, data in the package will be recorded in the corresponding array according to the format. After this step, processed data used in energy spectrums are passed to the drawing section, and the images are generated. To get hit position maps and LET spectrum, extra calculations are needed. Finally, the LET spectrum is generated. The images are generated by QChartView class. Methods for data analysis will be introduced in subsection 2.3. 


\subsubsection{Offline data analysis module}

Offline data analysis module shares the imaging part and most of the analysis part with data real-time imaging module, but is more efficient for data analysis and uses different data interface. It builds the data index as the first step and then analyzes the data.

\subsection{Data analysis}

Data analysis contains energy spectrum analysis, hit position calculation and LET calculation. These calculations are executed in different processes. The LET calculation uses the results of another two calculations.

\subsubsection{Energy spectrum analysis}

The values of data are ADC values, which can correspond to the energy deposited in the detector. The value of one channel is stored in an array, whose elements are the counts and their positions are the values. As the result of calibration, the electronics' LSB corresponds to electrons of $0.4 \mathrm{fC}$ for P-side and $0.35 \mathrm{fC}$ for $\mathrm{N}$-side. The energy spectrum shows the distribution of energy in a specific channel under a given gain.

\subsubsection{Hit position calculation}

The position of data in a package shows the channel it belongs to. There is a definite correspondence between channels and the strips of the detector. By counting the position of data, the hit positions of particles are recorded. Table 2 shows the possible situations.

Table 2: Situations of hit positions

\begin{tabular}{|c|c|c|c|c|}
\hline No. & Max hit num on a single layer & Triggered layers & Extra Info. & Validity \\
\hline 1 & 1 & 1 & $/$ & Invalid \\
\hline 2 & 1 & $\geq 2$ & $/$ & Valid \\
\hline 3 & 2 & 1 & $/$ & Invalid \\
\hline 4 & $\geq 2$ & $\geq 2$ & Adjacent & Valid \\
\hline 5 & $\geq 2$ & $\geq 2$ & Nonadjacent & Invalid \\
\hline
\end{tabular}

In situations 1 and 3, only one layer is triggered. Considering the energy of particles, rare probability can they be incident events in these two situations. Situation 5 is invalid because the is unable to determine the angle of incidence. The probability of multiple particles incident at the same time is very low so that few events are missed. In situations 1,3 , and 5 , the data will be discarded and won't be used in further processes. Situations 2 and 4 show single-particle incident events, and the difference is that the number of triggered channels is different due to the incident angle. In situation 2, the hit position is considered as the center of the corresponding strip. In situation 4, the hit position can be calculated by the Center-of-Gravity [8] of the strips. In situations 2 and 4, the results of the calculation will be used in LET calculation.

\subsubsection{LET calculation}

To calculate LET, it needs to confirm the species of particles. To achieve it, the $\Delta \mathrm{E}-\mathrm{E}$ method is used [9]. After that, three physical quantity is used to calculate LET. $\theta$ is the incident angle of a particle; $t$ is the thickness of one detector; $\mathrm{R}$ is the range of a stopping particle [10]. The range of protons and helium ions in silicon can be calculated [11][12].

Equations (1), (2), and (3) are used to calculate LET [13]. 


$$
\mathrm{LET}_{\text {Silicon }}=\frac{\mathrm{E}_{\mathrm{d}} \cos \theta}{\mathrm{t}}(1), \mathrm{LET}_{\text {Silicon }}=\frac{\mathrm{E}_{\mathrm{d}}}{\mathrm{R}}(2), \mathrm{L}_{\infty}=1.193 \times \mathrm{LET}_{\text {Silicon }} \text { (3). }
$$

Using position messages of at least two layers, which have been calculated in subsection 2.3.2, $\theta$ is determined. $t$ is given from the specification of the detectors. The energy of particles has been calculated in subsection 2.3.1. For particles passing through the detector, Equation (1) can be used to determine the LET. Equation (2) is used when particles stopped in the detector. For example, when the prototype uses three layers of detectors and two of them are triggered, the data of the first detector will be calculated by Equation (1), and the data of the second one will be calculated by Equation (2). Then the LET $_{\text {Silicon }}$ is used to determine $\mathrm{L}_{\infty}$ by Equation (3). After these calculations, the LET spectrum will be generated.

\subsection{Interface}

\subsubsection{Ethernet}

To communicate with the electronics through Ethernet, the Transmission Control Protocol (TCP) is used. The TCP is intended for use as a highly reliable host-to-host protocol between hosts in packet-switched computer communication networks, and in interconnected systems of such networks [14]. The DAQ software uses TCP as the protocol of Ethernet communication. The TCP communication class QtTcpSocket shows good performance and is widely used. So the software uses QtTcpSocket class to realize its function.

\subsubsection{USB 2.0}

USB makes the connection of peripheral devices to the computer easier and more efficient [15]. The microcontroller of the USB that is used on the electronics is CY7C68013A, which is a Cypress product. For good performance, CyUSB, which is a DLL provided by Cypress. is used to realize the function in the software.

\subsection{GUI}

GUI of the DAQ software is divided into 3 pages, according to the functions. The "Configuration" page includes basic configurations and commands, which are designed for configuring electronics and controlling data acquisition. The "Energy Spectrometer" page and "Hit Position" page are designed to show the corresponding images. Figure 4 shows the GUI of the software.

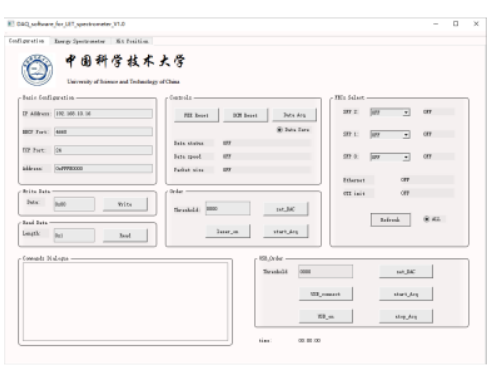

(a)

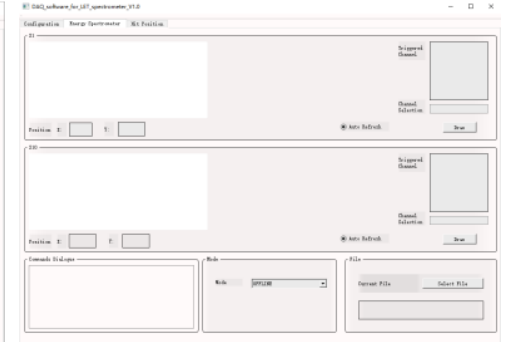

(b)

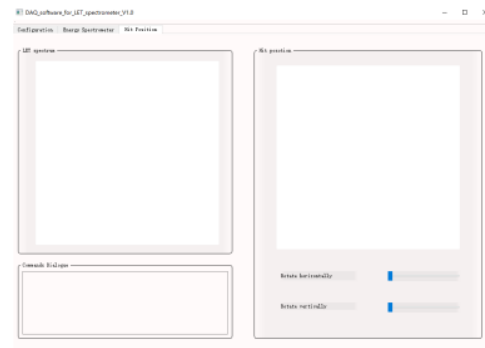

(c)

Figure 4: GUI of the software. (a) is the page of "Configuration"; (b) is the page of "Energy Spectrometer"; (c) is the page of "Hit Position". 


\section{A Preliminary Test}

To evaluate the correctness of the DAQ software, an ${ }^{241} \mathrm{Am}$ radioactive sample and the prototype are used to test the software. Only one detector is used in the test. To simulate the environment in space, the sample and prototype are set into a vacuum tank (pressure $10 \mathrm{~Pa}$ ). The upper computer is connected with the prototype through USB 2.0. The software is run on Windows 10 platform. The upper computer has an Intel Core i5-4570 CPU with 8 GB RAM. Some test results are shown in Figure 5. The range of low gain channels is 0.3-50 MeV with lower resolution. The range of high gain channels is $0.13-10 \mathrm{MeV}$ with higher resolution.

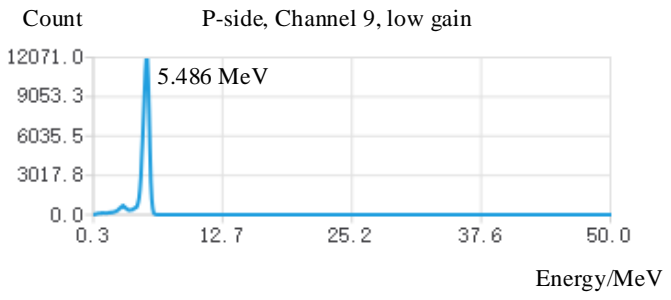

(a)

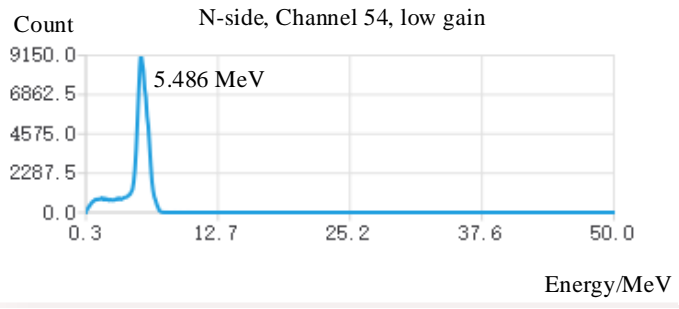

(c)

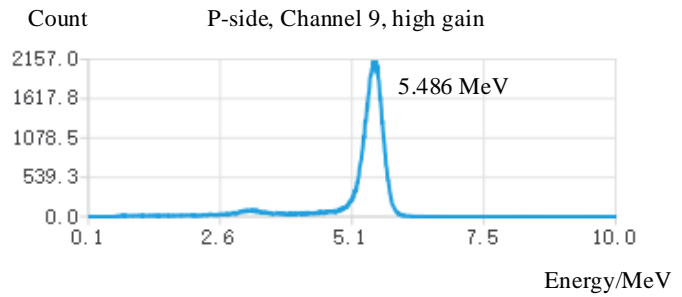

(b)



(d)

Figure 5: Some results of the preliminary test. (a) is the energy spectrum based on the data of channel 9, which has low gain and from the P-side of the detector. (b) is the energy spectrum of channel 9's data of high gain. (c) is the energy spectrum based on the data of channel 54, which has low gain and from the Nside of the detector. (d) is the energy spectrum of channel 54's data of high gain.

As is shown in these figures, the peak corresponds to the energy of $5.486 \mathrm{MeV}$ is generated by helium ions emitted by the ${ }^{241} \mathrm{Am}$ sample. To obtain higher resolution, further analysis will be carried out with the data of high gain. The counts on the left of the peak are generated by the particles that hit the gap of the strips because the energy in one incident event is deposited by the two adjacent strips. The results show that the DAQ software successfully reads out and analyzes the data from the prototype, and fits the needs of application.

\section{Conclusions}

ADAQ software for a prototype of LET spectrometer is proposed in this paper. The structure of the software is modularized. Real-time analysis and LET calculation are supported in the software. The test results show the software meets the needs of application.

\section{Acknowledgments}

This work is supported by the State Key Laboratory of Particle Detection and Electronics, SKLPDE-ZZ-202104. 


\section{References}

[1] Adams, J., Falconer, D., \& Fry, D. (2012, November). The ionizing radiation environment in space and its effects. In AIP Conference Proceedings (Vol. 1500, No. 1, pp. 198-203). American Institute of Physics.

[2] Takahashi, A., Ikeda, H., \& Yoshida, Y. (2018). Role of high-linear energy transfer radiobiology in space radiation exposure risks. International journal of particle therapy, 5(1), 151-159.

[3] Rossi, H. H. (1980). Estimates of the hazard of low doses of ionizing radiation. IEEE Transactions on Nuclear Science, (1), 23-27.

[4] Qt Company. https://www.qt.io/product/framework

[5] Microsoft Corporation. https://dotnet.microsoft.com/apps/aspnet/mvc

[6] Bray, T., Paoli, J., Sperberg-McQueen, C. M., Maler, E., \& Yergeau, F. (1997). Extensible markup language (XML). World Wide Web Journal, 2(4), 27-66.

[7] Tubishat, M., Alsmadi, I., \& Al-Kabi, M. (2009, March). Using XML files to document the user interfaces of applications. In 2009 5th IEEE GCC Conference \& Exhibition (pp. 1-4). IEEE.

[8] A. Tykhonov, et al., "Internal alignment and position resolution of the silicon tracker of DAMPE determined with orbit data," Nuclear Instruments and Methods in Physics Research Section A: Accelerators, Spectrometers, Detectors and Associated Equipment, vol. 893, pp. 43-56, 2018.

[9] Carboni, S., Barlini, S., Bardelli, L., Le Neindre, N., Bini, M., Borderie, B., ... \& Fazia Collaboration. (2012). Particle identification using the $\Delta \mathrm{E}-\mathrm{E}$ technique and pulse shape discrimination with the silicon detectors of the FAZIA project. Nuclear Instruments and Methods in Physics Research Section A: Accelerators, Spectrometers, Detectors and Associated Equipment, 664(1), 251-263.

[10] Terasawa, K., Borak, T. B., Doke, T., Fuse, T., Hara, K. I., Kikuchi, J., ... \& Uchihori, Y. (2005). The response of the silicon-based linear energy transfer spectrometer (RRMD-III) to protons from 1 to 70 MeV. Japanese journal of applied physics, 44(10R), 7608.

[11] Tykhonov, A., Ambrosi, G., Asfandiyarov, R., Azzarello, P., Bernardini, P., Bertucci, B., ... \& Zimmer, S. (2018). Internal alignment and position resolution of the silicon tracker of DAMPE determined with orbit data. Nuclear Instruments and Methods in Physics Research Section A: Accelerators, Spectrometers, Detectors and Associated Equipment, 893, 43-56.

[12] Bragg, W. H., \& Kleeman, R. (1905). XXXIX. On the $\alpha$ particles of radium, and their loss of range in passing through various atoms and molecules. The London, Edinburgh, and Dublin Philosophical Magazine and Journal of Science, 10(57), 318-340.

[13] Sakaguchi, T., Doke, T., Hasebe, N., Hayashi, T., Kashiwagi, T., Kikuchi, J., ... \& Takahashi, S. (1999). LET distribution measurement with a new real-time radiation monitoring device-III onboard the Space Shuttle STS-84. Nuclear Instruments and Methods in Physics Research Section A: Accelerators, Spectrometers, Detectors and Associated Equipment, 437(1), 75-87.

[14] Postel, J. (1981). Transmission control protocol.

[15] Ramadoss, L., \& Hung, J. Y. (2008, November). A study on universal serial bus latency in a real-time control system. In 2008 34th Annual Conference of IEEE Industrial Electronics (pp. 67-72). IEEE. 